\title{
El procedimiento administrativo sancionatorio en el marco de las empresas de servicios públicos domiciliarios: aproximación teórica a sus postulados básicos*
}

\author{
Fanny Gineth Castro Dulcey* \\ Jorge Rafael Gómez Ortiz
}

Recepción: 20 de marzo de 2020 • Aprobación: 10 de mayo de 2020

\section{Resumen}

La Superintendencia de Servicios Públicos Domiciliarios se encarga de la inspección, vigilancia y control de las empresas de servicios públicos domiciliarios. Dentro de ese contexto, dicha autoridad administrativa tiene la facultad de imponer sanciones a tales prestadoras, para lo cual se sirve del procedimiento administrativo sancionatorio general consagrado en la Ley 1437 de 2011 en concordancia con otras normas de orden sustancial dispersas en el ordenamiento normativo nacional, que determinan

\footnotetext{
El presente documento es producto del proyecto de investigación "El procedimiento administrativo sancionatorio en el marco de las empresas de servicios públicos domiciliarios. Aproximación teórica a sus postulados básicos", gestionado en el programa de la Maestría en Derecho Administrativo gestionado en la Universidad Libre, Seccional Bogotá, Colombia.

Citar como: Castro Dulcey, F. G. y Gómez Ortiz, J. R. (2020). El procedimiento administrativo sancionatorio en el marco de las empresas de servicios públicos domiciliarios. Aproximación teórica a sus postulados básicos. Revista IUSTA, 53,161-188. DoI: https://doi.org/10.15332/25005286.6275

"* Abogada, egresada de la Corporación Universitaria Republicana, especialista en Derecho Público, y Derecho Procesal Constitucional de la misma institución, con especialización en Derecho Administrativo de la Universidad Libre de Colombia. Candidata a magíster en Derecho Administrativo de la Universidad Libre de Colombia. Profesional de la Empresa de Acueducto y Alcantarillado de Bogotá. Correo electrónico: fannygcastrod@gmail.com. oRcid: 0000-0002-0638-2367.

Abogado, especialista en Derecho Administrativo y en Derecho Constitucional de la Universidad Libre de Colombia. Candidato a magíster en Derecho Administrativo de la misma institución. Profesional Especializado del Consejo de Estado. Correo electrónico: Jorgerafael88@hotmail.com. ORCID: 0000-0003-3156-2025.
} 
las sanciones por imponer, pero especialmente los criterios bajo los cuales ha de ejercerse dicha potestad. Sin embargo, no existe claridad suficiente en relación con si la forma en la que están dispuestos estos instrumentos jurídicos realmente conduce al cumplimiento de los postulados básicos de cualquier procedimiento administrativo sancionatorio, esto es, si se garantiza el principio de legalidad, la tipicidad, la oportunidad, la gradualidad, el derecho de defensa, el non bis in idem y otras garantías inherentes al debido proceso que debe preceder a la imposición de las sanciones que pueden llegar a imponerse a las empresas de servicios públicos domiciliarios. Y es dentro de ese contexto que el presente artículo ofrece una aproximación a los elementos jurídicos y teóricos que permiten definir si el procedimiento administrativo sancionatorio en cuestión satisface los postulados básicos que deben ser inherentes a este tipo de actuaciones.

Palabras clave: procedimiento administrativo sancionatorio, Superintendencia de Servicios Públicos Domiciliarios, empresas de servicios públicos domiciliarios, debido proceso, derecho de defensa.

\title{
The Administrative Sanctioning Procedure within the Framework of Domestic Utilities Companies: A Theoretical Approach to its Basic Postulates
}

\begin{abstract}
The Superintendency of Domestic Utilities is responsible for the inspection, surveillance, and control of domestic utilities companies. Within this context, this administrative authority has the power to impose sanctions on such providers, for which it uses the general administrative sanction procedure enshrined in Law 1437 of 2011, in accordance with other rules of substantial order dispersed in the national regulatory system, which determine the sanctions to be imposed, but especially the criteria under which said power must be exercised. However, there is insufficient clarity as to whether the way in which these legal instruments are laid down actually leads to the fulfilment of the basic tenets of any administrative sanctioning procedure, that is, whether the principle of legality, typicity, timeliness, gradualness, the right of legal defense, the non bis in idem and other guarantees inherent in the due process that must precede the imposition of the sanctions that may be imposed on domestic utilities, are actually guaranteed. And it is within this context that this article offers an approximation to the legal and theoretical elements
\end{abstract}


that make it possible to define whether the administrative sanctioning procedure in question satisfies the basic postulates that must be inherent in this type of action.

Keywords: administrative sanctioning procedure, Superintendency of Domestic Utilities, domestic utilities companies, due process, right of legal defense.

\section{O PROCEDIMENTO ADMINISTRATIVO PUNITIVO NO ÂMBITO DAS EMPRESAS DE SERVIÇOS PÚBLICOS DOMICILIARES: APROXIMAÇÃo TEÓRICA DE SEUS PRINCÍPIOS BÁSICOS}

\section{Resumo}

A Superintendência de Serviços Públicos Domiciliares é responsável pela inspeção, pela vigilância e pelo controle das empresas de serviços públicos domiciliares. Nesse contexto, essa autoridade administrativa tem a faculdade de impor sanções a essas prestadoras utilizando o procedimento administrativo punitivo geral consagrado na Lei 1.437 de 2011 em concordância com outras normas de ordem substancial dispersas no ordenamento normativo nacional, que determinam as sanções a impor, mas especialmente os critérios sob os quais esse poder pode ser exercido. Contudo, não há clareza suficiente sobre se a forma na qual estão dispostos esses instrumentos jurídicos realmente conduz ao cumprimento dos princípios básicos de qualquer procedimento administrativo punitivo, isto é, se são garantidos o princípio de legalidade, a tipicidade, a oportunidade, a gradualidade, o direito de defesa, o el non bis in idem e outras garantias inerentes ao devido processo que deve preceder à imposição das sanções das empresas de serviços públicos domiciliares. Nesse sentido, este artigo oferece uma aproximação dos elementos jurídicos e teóricos que permitem definir se o procedimento administrativo punitivo em questão satisfaz os princípios básicos que devem ser inerentes a esse tipo de atos.

Palavras-chave: procedimento administrativo punitivo, Superintendência de Serviços Públicos Domiciliares, empresas de serviços públicos domiciliares, devido processo, direito de defesa. 


\section{Introducción}

Determinar si el régimen administrativo sancionatorio consagrado en la Ley 1437 de 2011, aplicado a las empresas de servicios públicos domiciliarios, permite el cumplimiento de los postulados base de un procedimiento administrativo sancionatorio implica transitar por el sendero demarcado por la Constitución Política, las leyes, la jurisprudencia y la doctrina que atañe a dicha problemática, extrayendo de tales fuentes insumos jurídicos y teóricos que permiten establecer por lo menos cuatro diferentes entradas al asunto.

Antes que demostrar cuál de ellas es la que mejor se aviene al contexto colombiano, resulta conveniente, en primera medida, poner en evidencia si la normatividad vigente es suficiente en el tema bajo estudio y cuál es el aporte de la jurisdicción en materia de insumos jurídicos que pudieran suplir las eventuales falencias de la norma, desde un plano abstracto y meramente teórico.

Una primera mirada supone que las normas del procedimiento administrativo sancionatorio en cuestión permiten en sí mismas satisfacer la necesidad de que se respeten al máximo las garantías de las empresas enjuiciadas por la Superintendencia de Servicios Públicos Domiciliarios, en la medida en que cualquier vacío normativo tiene que ser dimensionado a partir de la nomoárquica que tiene por fuente directa a la Constitución Política, de suerte que por vía de principios constitucionales se supla cualquier carencia legislativa.

Otra forma de entenderlo, en cambio, sería que al estar plagado de ambigüedades y vaguedades el régimen sancionatorio dispuesto para las empresas de servicios públicos domiciliarios resulta inaplicable, comoquiera que por ser una expresión del ius puniendi se debe propender por el máximo apego a la legalidad, lo que de suyo implica la imposibilidad de suponer la existencia de contenidos que no respondan a la literalidad de la norma.

Aunque, en este punto, debe decirse que tales enfoques no necesariamente tendrían que ser excluyentes, dado que bajo una suerte de comprensión mixta cabría entender que, para que se entiendan garantizados los postulados de un verdadero procedimiento administrativo sancionatorio, basta la existencia de unos contenidos mínimos normativos, que puedan ser llenados con otro tipo de previsiones, a la manera de tipos en blanco cuyo alcance dependa de la integración con otras pautas de mayor especificidad. 
Finalmente, aunque sin pretender agotar todos los escenarios, podría abordarse el tema de la aplicación de los postulados en cuestión bajo la égida de que se trata de una construcción dinámica en la que el activismo judicial tiene un papel protagónico, en el entendido de que el derecho se depura y complementa desde la jurisprudencia; por ende, de haber una disyuntiva sobre el parámetro normativo definitorio de la regla sancionatoria aplicable en vía administrativa, debe ser dirimida por el juez - unipersonal o colegiado-, que con el solo pronunciamiento habrá trazado una senda que guíe la resolución de casos futuros por parte de la Superintendencia de Servicios Públicos Domiciliarios.

La calificación de cualquiera de esos escenarios como el más acertado y aproximado para describir la realidad frente a la aplicación de los condignos postulados base, desde la senda procesal demarcada por la Ley 1437 de 2011, depende del estándar de exigencia regulatoria que se pretenda y de las garantías o referentes que, en sí mismos, deban tomarse como postulados para satisfacer ese propósito. No obstante, cualquiera que sea el caso, resulta conveniente por lo menos referenciar algunos de los que podrían avenirse a tal categorización apelando a niveles normativos, jurisprudenciales y doctrinales, como los que se esbozan en lo sucesivo del presente artículo.

\section{Aproximación al contexto de la potestad sancionatoria del Estado}

Si bien el derecho sancionatorio se inscribe en el ius puniendi del Estado, según Restrepo y Nieto (2017), se trata en todo caso de una disciplina compleja que se traduce en un género que comporta, al menos, cinco especies, a saber: el derecho penal delictivo, el derecho contravencional, el derecho disciplinario, el derecho correccional y el derecho de punición o por indignidad política, también conocido como impeachment.

Dicho régimen punitivo es de vital importancia como herramienta para el cumplimiento de los fines del Estado, consagrados principalmente en el artículo $2^{\circ}$ de la Constitución Política, al cual subyace la prestación de los servicios públicos domiciliarios, que por su valor estratégico y social demanda de la supervisión, vigilancia y control que recae sobre las empresas a las que se les ha confiado su prestación (Millán, Blanco y Guecha, 2017). 
Es por ello que, como desarrollo de estas máximas, se debe fijar especial cuidado a la llamada potestad sancionadora que ejerce la administración frente a los particulares e inclusive sobre ciertas autoridades públicas, la cual ha sido definida por la Corte Constitucional y el Consejo de Estado como "la capacidad de imponer castigos o sanciones correctivas para el logro del interés general" (C.E., Sentencia 1996-00680/20738), "constituyéndose como un complemento de la potestad de mando, pues contribuye a asegurar el cumplimiento de las decisiones administrativas" (C.C., Sentencia C-214, 1994).

Es evidente que esta potestad sancionatoria, en el caso que ocupa el presente documento, es ejercida por la Superintendencia de Servicios Públicos Domiciliarios y tiene, en clave de debido proceso, el desarrollo de facultades administrativas de control y no jurisdiccionales, tema que no admite mayor discusión en el ordenamiento colombiano (Guarín, 2015).

Es claro que esta facultad debe tener limitaciones, aunque, en principio, no tendría por qué resultar clara la intensidad de estas. Se trata de un problema cuya solución está estrechamente ligada a la ubicación dogmática de la rama que las estudia, entre las que refulgen dos posibilidades: en el derecho penal o en el derecho administrativo (Cordero, 2009). En concordancia con ello, se debe reiterar que este último es una manifestación de un mismo y único ius puniendi del Estado, pero a falta de precisos principios en su aplicación debieran considerarse la extrapolación de tales referentes por partes de sistemas normativos de similar naturaleza, por urgencia y por no haber otros principios en este orden de materias con tal grado de desarrollo con excepciones o con matices, según corresponda, pero sin llegar en estricto sentido al rigor de lo penal (Cordero, 2009), por ello es que ha de llamarse derecho administrativo sancionador y no derecho penal administrativo (Aldana y Guarín, 2016).

De tal manera, la facultad sancionadora de la administración se diferencia de la penal, pues busca que no se quiebren los deberes que tienen los administrados con la administración; de ahí que la multa es la sanción más emblemática y nunca la privación de la libertad, aunado a que sus actos pueden ser revisados por la jurisdicción (Ramírez y Aníbal, 2015).

De ahí que se entienda que el derecho administrativo sancionador nace como disciplina independiente, pero que sigue, en mínimos aspectos, los principios del derecho penal y que no se desarrolla con fundamento en principios exclusivamente 
propios, por cuanto no existe una legislación específica que lo regule con exhaustividad (De La Cruz, 2018).

Ahora, si bien el derecho administrativo sancionatorio ha sido asimilado en varios aspectos con el derecho penal y sus garantías, también es importante aclarar que la Corte Constitucional ha manifestado, a propósito de su diferenciación, que existen hechos que deben ser objeto de sanción directa de la administración, bajo otro tipo de consideraciones (C.C., Sentencia C-214/94).

De este modo, el derecho administrativo sancionador, en términos de la doctrina generalizada y la jurisprudencia constitucional, supone una ruptura del principio clásico de la tridivisión de poderes, en la medida en que la represión de los ilícitos ya no corresponde de manera exclusiva al poder judicial, y más concretamente a la justicia penal. En efecto, el modelo absoluto de separación de funciones del poder público se reveló como insuficiente ante el incremento de deberes y obligaciones de los particulares, como de funciones públicas de los servidores del Estado, que ante su incumplimiento merecían la imposición de una sanción. Sin embargo, no todas las infracciones eran susceptibles del mismo tratamiento, pues en atención a los intereses que se pretendían proteger con cada una las disciplinas del derecho punitivo del Estado se distinguieron aquellas que serían objeto de sanción directa por la Administración, y aquellas otras que se reservarían para la justicia penal (C.C., Sentencia C-818, 2005).

Por lo tanto, en la aplicación del procedimiento administrativo sancionatorio se deben tener en cuenta principios básicos aplicados al derecho penal (Mesa, 2016) que cobran una importancia clara en la garantía de derechos fundamentales como el debido proceso (Pardo, 2014).

Por otro lado, es menester tomar en consideración lo consagrado en la Ley 1437 de 2011, en cuanto al procedimiento administrativo sancionatorio general dispuesto en los artículos 47 al 52, de cara a la necesidad de unificar criterios acerca de principios aplicables a dichos procedimientos y etapas del mismo; lo cual contrasta con la pervivencia de procedimientos sancionatorios especiales, que, según un estudio publicado por la universidad Eafit, entre 1991 y 2011, bordeaban la escandalosa cifra de 97 regulaciones de ese tipo (Tamayo, Mejía y Restrepo, 2014); circunstancia que obliga a evaluar cómo ha sido la aplicación de dicho procedimiento en materia de servicios públicos domiciliarios; ámbito que, paradójicamente, carece de un procedimiento administrativo especial (Cortes, 2013). 
En línea con lo anterior, ha de señalarse que en la actualidad sigue existiendo una dispersión normativa en lo referente a las conductas constitutivas de sanción administrativa. Ello hace que los administrados y las entidades administrativas tengan dificultades para dimensionar los comportamientos proscritos por el ordenamiento jurídico y el trámite que se debe surtir para la aplicación de la consecuencia respectiva, a lo cual no es ajena la Superintendencia de Servicios Públicos domiciliarios como órgano encargado de aplicar tal procedimiento en el ámbito de los asuntos de su competencia.

Esto implica que las consecuencias adjetivas y sustantivas que la mencionada autoridad hace recaer sobre las empresas de servicios públicos las afectan al no estar previamente establecidos en su totalidad, al punto en que deben ser contrastados con las limitaciones impuestas jurisprudencialmente frente al poder sancionatorio en cuestión.

Es por ello que no resulta extraño hablar de las falencias que persisten a la expedición de la Ley 1437 de 2011 sobre la materia, especialmente frente a los eximentes de responsabilidad frente a los cuales se refirió Laverde en videoconferencia ante el Consejo de Estado (2016). A esto se suma la falta de regulación legal que impide su ejercicio en ciertos eventos, así como posibles debilidades en materia de tipicidad de la infracción o de la gradualidad del correctivo aplicado en el contexto de los servicios públicos domiciliarios, que tienen como hecho indicador de que muchas de estas disputas derivadas de tales falencias posiblemente no encuentran solución pronta y efectiva en las vías ordinarias, los numerosos casos en que se acude a la acción de tutela con miras a conjurar la vulneración de derechos fundamentales, efecto que, de otra manera, sería difícil de conseguir con el mismo grado de satisfacción.

En materia de sanciones, ha sido en múltiples ocasiones criticado el monto pecuniario que entrañan, por lo que es importante observar en el marco de estas actuaciones el principio de proporcionalidad, que, si bien no está plasmado en la Ley 1437 de 2011, no por ello debe desconocerse en una actuación, pues hace parte de la reclamada gradualidad de la sanción. Si una decisión jurídica (como la de imponer o no una sanción administrativa) ha de ser racional, entonces debe efectuar una ponderación de los intereses y derechos en conflicto (León, 2009).

No se puede olvidar que el ejercicio de la actividad sancionatoria siempre debe guardar relación con el carácter preventivo que resulta de su ejercicio, pues con ello se busca salvaguardar los bienes jurídicos protegidos y mejorar la prestación del 
servicio; de manera que la Superintendencia debe ejercer sus funciones de vigilancia y control para que los prestadores cumplan cabalmente las obligaciones que las normas del régimen de servicios públicos domiciliarios les imponen respecto de los usuarios (Sierra, 2015); eso sí, respetando los límites que el propio ordenamiento le impone, así ello suponga recabar en valores, principios y reglas dispersos en el universo jurídico en que se inscribe.

Frente a ello, el escenario ideal devendría de disminuir la discrecionalidad desbordada y la arbitrariedad frente a las etapas, términos y demás pormenores del procedimiento que se adelanta para culminar la actividad sancionatoria (Tamayo, Mejía y Restrepo, 2014).

Bajo esa égida, tal y como se ha venido anunciado, tomando como referente temporal la promulgación de la Constitución Política de 1991, es dable señalar que el procedimiento administrativo sancionatorio aplicado a las empresas de servicios públicos domiciliarios en Colombia ha sido abordado desde diferentes ámbitos, entre ellos: 1) el normativo, como fruto del ejercicio de la actividad legislativa y de la potestad regulatoria que el Texto Fundamental, referente en sí mismo, confiere al Gobierno Nacional; 2) el jurisprudencial, que viene dado por la labor hermenéutica que los operadores jurisdiccionales imprimen a tales premisas, especialmente la Corte Constitucional y el Consejo de Estado, como órganos límites en materia de control de constitucionalidad (abstracto y concreto) y de legalidad, respectivamente; 3) y el doctrinal, que recoge la visión de ciertos estudiosos del derecho que se han dado a la tarea, de un lado, de poner en contexto la confrontación entre las reglas de derecho sobre la materia y la realidad fáctica o material que a ellas subyace, y del otro, de observar con visión crítica la validez jurídica de tales postulados.

\section{Aproximación normativa (constitucional, legal y reglamentaria) a los postulados del procedimiento administrativo sancionatorio aplicado a las empresas de servicios públicos domiciliarios}

Bajo esta perspectiva, el principal referente es la Constitución Política de 1991, comoquiera que a partir de ella surgen las bases de todo el modelo de prestación de servicios públicos en Colombia, y desde luego los principios que orientan la 
prestación de estos por parte de las empresas, tanto del sector público como del privado, que han sido habilitadas para desempeñar este rol.

En tal sentido, el preámbulo sostiene que el Pacto Político de 1991 pretende, entre muchos otros fines, garantizar un orden político económico y social justo, lo cual se armoniza con lo consignado en el artículo $1 .^{\circ}$, que establece como uno de sus principales fundamentos el respeto de la dignidad humana, y el artículo 2. ${ }^{\circ}$, en el que cobra relevancia el servicio a la comunidad como un fin esencial del Estado, cuyas autoridades han sido instituidas, según lo prevé la presente disposición, para asegurar el cumplimiento de los deberes propios, pero también los que conciernen a los particulares, dentro de la perspectiva incesante de mantener la vigencia de un orden social justo.

Bajo la misma línea destacan las reglas fijadas en los artículos 356 y 357 de la Constitución Política de 1991, en tanto establecen una partida de destinación específica del Sistema General de Participaciones de los departamentos, distritos y municipios, con destino a la financiación de los servicios públicos domiciliarios de agua potable y saneamiento básico, precisando además a los referidos entes territoriales deberes en términos de cobertura universal y estándares de calidad fijados por las autoridades competentes.

$\mathrm{Y}$ aunque en estos preceptos no se mencionan otras categorías o tipos de servicios públicos domiciliarios, sí se hacen importantes precisiones dentro del título XII de la Carta, "Del régimen económico y de la hacienda pública", capítulo 5, "De la finalidad social del Estado y de los servicios públicos”, entre los artículos 365 y 366 de tales acápites.

Allí se estipula de forma expresa que los servicios públicos son inherentes a la finalidad social del Estado, al que se le confía la función de mantener la regulación, el control y la vigilancia de dichos servicios.

De manera concreta, frente a los denominados servicios públicos domiciliarios, el artículo 367 superior establece una reserva legal, en cuanto dispone que "la ley" fijará las competencias y responsabilidades relativas a su prestación — la cual atribuye de forma directa a los municipios, con apoyo y coordinación de los departamentos-, cobertura, calidad, financiación y el régimen tarifario - el cual tendrá en cuenta criterios de costos, solidaridad y redistribución de ingresos.

Bajo la misma reserva, a las voces de lo normado en el artículo 368 de la Carta, quedó cobijada la determinación de los derechos y deberes de los usuarios y la forma 
en la que se lleva a cabo, entre otros aspectos, la fiscalización de las empresas que participan del anotado modelo prestacional.

Destacado el anterior contexto constitucional en el que se enmarca el tema que concita el presente artículo, resta señalar que, de manera, expresa, puntual y precisa, el artículo 370 de la Norma Normarum consagra las bases orgánicas y funcionales de la potestad sancionatoria que se ejerce en relación con las empresas de servicios públicos domiciliaros, en tanto se define que "corresponde al Presidente de la República señalar, con sujeción a la ley, las políticas generales de administración y control de eficiencia de los servicios públicos domiciliarios y ejercer, por medio de la Superintendencia de Servicios Públicos Domiciliarios, el control, la inspección y vigilancia de las entidades que los presten".

Bien puede decirse, entonces, que con la Constitución de 1991 se permitió que personas de derecho público, privado, o mixtas pudieran prestar servicios públicos domiciliarios; se autorizaron, a los municipios, a las entidades descentralizadas, a las organizaciones comunitarias y a las personas naturales o jurídicas para tal efecto. No obstante, está claro que el Estado se reservó la facultad de regular, controlar y vigilar a los empresarios de dicha actividad (Ramírez y Echeverría 2009), sin desconocer que este nuevo pacto político también permitió la participación de los usuarios en la gestión y seguimiento a las actividades desarrolladas por los prestadores de servicios públicos.

En cuanto concierne al plano legislativo, cabe decir que existe un Régimen de los Servicios Públicos Domiciliarios, contenido en la Ley 142 de 1994 y las normas que lo modifican, dentro del cual se da forma a los aspectos esenciales en materia de prestación a cargo de las empresas habilitadas para tal efecto, pero, sobre todo, en punto a las consecuencias derivadas de su transgresión, lo cual comprende la manera en la que estas últimas llegan a imponerse y los medios para hacer efectivas las determinaciones en torno a ellas. No obstante, hay quienes consideran que la Ley 142 de 1994 amerita una nueva regulación, actualizada con las diferentes normas de procedimiento general que han sido emitidas desde 1994, al estimar también que esta ley aún es restrictiva en cuanto a los postulados del debido proceso y garantías de los usuarios, entre otros aspectos.

Así, un primer acercamiento al tema permite entrever que el artículo $6^{\circ}$ del apuntado referente legal faculta al superintendente de Servicios Públicos Domiciliarios para imponer sanciones, incluso a los alcaldes distritales y municipales y a los gobernadores departamentales para intervenir empresas de servicios públicos 
domiciliarios y disponer de bienes de las entidades territoriales para que se cumpla a cabalidad con el cometido constitucional que les concierne.

El artículo 11 de la ley citada recalca la función social de la propiedad en las entidades prestadoras de servicios públicos, a las que se hace civilmente responsables por los perjuicios ocasionados a los usuarios, con lo cual se va demarcando, desde ya, el radio de acción dentro del cual debe moverse la Superintendencia, que es el órgano por excelencia encargado de aplicar las sanciones administrativas por la violación del régimen prestacional en cuestión, claro está, dentro del marco de atribuciones delimitado por los artículos 79 y 80 de esta normativa, que detallan el ejercicio de la inspección, vigilancia y control que se le encomienda a la entidad en general y a la autoridad que la regenta en particular, incluida la facultad de imponer sanciones.

Otro aspecto cardinal deviene de la coordinación con otras autoridades, entre las que destaca la Comisión de Regulación, que, en los términos del artículo 73 de la Ley 142 de 1994, despliega su potestad regulatoria frente a posibles monopolios en el sector, y que, además, tiene capacidad para instar la apertura de investigaciones administrativas ante la autoridad competente.

Por su parte, el artículo 81 contiene todo el catálogo de las sanciones que la Superintendencia de Servicios Públicos Domiciliarios puede imponer a quienes violen las normas a las que se sujetan, que comprende, entre otras medidas, amonestaciones, multas, suspensiones, prohibiciones e intervenciones. En relación con esta cláusula normativa, destacan las modificaciones realizadas por el artículo 208 de la Ley 1753 de 2015, contentiva del Plan Nacional de Desarrollo de su correspondiente vigencia, los cuales no se abordarán detalladamente en el presente acápite, por no ser de su objeto, pero que se deben mencionar por constituir un referente ineludible del estudio, según se verá más adelante, en tanto determinan parámetros específicos para las eventuales sanciones.

Cabe mencionar que la Ley 142 de 1994 establece también otro tipo de obligaciones sancionables, relacionadas con la estratificación de los municipios, la asignación de subsidios, el recaudo de gravámenes para el sector energético, de acueducto y gas, entre otros, que no recaen directamente sobre las empresas de servicios públicos domiciliarios, sino sobre otro tipo de actores, y que si bien no constituyen un referente directo en cuanto al tema objeto del presente esbozo, desde el punto de vista comparativo representan un insumo al que se puede acudir en aras de confrontar parámetros de proporcionalidad, temporalidad y otras características 
útiles en el estudio del procedimiento administrativo sancionatorio aplicado a las prestadoras del servicio.

Este espectro regulatorio ha sido objeto de reglamentación por parte del Gobierno Nacional, mediante disposiciones de tipo universal, impersonal y abstracto que dan contenido específico a los ejes del derecho administrativo sancionatorio aplicado a las empresas de servicios públicos domiciliarios.

Entre la literatura jurídica que integra este segmento de fuentes de información sobre la materia destaca el Decreto 1077 de 2015, por medio del cual se expide el decreto único reglamentario del sector vivienda, ciudad y territorio vigente, en cuya parte 3 se delinean aspectos relacionados con el régimen reglamentario del sector agua potable y saneamiento básico.

Allí se aborda lo concerniente al trámite que debe surtirse ante la Superintendencia de Servicios Públicos Domiciliarios para hacer cumplir a las prestadoras, incluso por vía de sanciones, sus obligaciones frente a la disponibilidad inmediata del servicio en la ejecución de proyectos de vivienda, la prohibición de requisitos adicionales y la distribución de responsabilidades entre estas y los urbanizadores o constructores, y las consecuencias de la desatención de sus deberes frente a los usuarios.

En el Decreto 1082 de 2015, "por medio del cual se expide el decreto único reglamentario del sector administrativo de planeación nacional”, se encuentran los criterios y la metodología para graduar y calcular las multas por imponer, así como las circunstancias de atenuación, por parte de la Superintendencia de Servicios Públicos Domiciliarios con ocasión de infracciones relacionadas con los servicios de energía eléctrica; acueducto, alcantarillado y aseo; y gas combustible.

Con orientación similar se destacan las modificaciones introducidas por los decretos 1082 de 2015, 281 de 2017 y 1158 de 2017 al régimen sancionatorio enunciado en precedencia, y que se han visto afectados, de una u otra forma, por las distintas sentencias de constitucionalidad que se han producido sobre la materia, algunas directamente sobre la legislación reglamentada por tales decretos, según se verá más adelante. 


\section{Aproximación desde el ámbito jurisprudencial a los postulados del procedimiento administrativo sancionatorio aplicado a las empresas de servicios públicos domiciliarios}

Otro de los escenarios desde el cual ha sido abordado el régimen sancionatorio aplicado a las empresas de servicios públicos domiciliarios, sin duda, es el de la actividad productora de las altas Cortes, principalmente la Corte Constitucional y el Consejo de Estado, en cuyas providencias se decanta un nutrido análisis sobre la comprensión de aspectos cardinales, en relación con el cumplimiento de las formas que deben cumplirse en el marco del debido proceso que conduce a la imposición de eventuales sanciones, así como desde el estudio de los elementos sustanciales, definidos por la nomoárquica y la interpretación de los diferentes ingredientes normativos en los que se inscribe tal consecuencia jurídica - legalidad, proporcionalidad, razonabilidad, etcétera-.

Si bien el régimen de servicios públicos de Colombia corresponde al nuevo Estado social de derecho como modelo y principio fundante, así como la libertad económica sobre la cual se postula el sistema; dentro de la Ley 142 de 1994 muchas cosas no quedaron claras por el legislador; no obstante, el apoyo que han brindado los conceptos jurídicos emitidos por la Superintendencia y las sentencias emitidas por la Corte Constitucional han propendido por restablecer la armonía de los preceptos normativos que definen el régimen jurídico de los SPD, con aquellos principios constitucionales y legales que lo fundamentan (Arocha, 2013), permitiendo de esta manera aclarar jurídicamente el contenido de algunas disposiciones legales que se tornan difusas, así como eliminar y complementar algunas otras. Por ello, al momento de estudiar su ámbito jurídico se debe no solo tener en cuentas el marco jurídico, sino aquellos aspectos conceptuales que complementan la forma de interpretación de la norma.

Desde el plano de la jurisprudencia de la guardiana de la supremacía e integridad de la Constitución Política se resalta la Sentencia C-092 de 2018, que realiza aportes sobre la facultad de imposición de sanciones a particulares que prestan servicios públicos domiciliarios, la legitimidad en el ejercicio de la potestad reglamentaria del Gobierno Nacional en la materia, junto con las tensiones entre este 
régimen sancionatorio y la libertad de empresa aplicada a la prestación de este tipo de servicios, entre otros aspectos.

Igualmente, en la Sentencia su-1010 de 2008 la Corte Constitucional examinó la imposición de sanciones por parte de empresas de servicios públicos domiciliarios a sus usuarios como una infracción de las obligaciones constitucionales y legales de estas prestadoras, determinando su prohibición.

Por su parte, en la Sentencia C-558 de 2001, la alta Corporación se refirió al ejercicio de la función administrativa por empresas de servicios públicos, a la naturaleza de los actos emitidos por estas, a los derechos y prerrogativas de la autoridad pública frente a ellas, a las características del contrato de servicios públicos y su carácter de fuente de derechos y obligaciones.

Así también, la Sentencia C-595 de 2010 sienta importantes bases en relación con la naturaleza objetiva del derecho sancionador. Cabe decir que en este caso se refirió a una norma de orden ambiental, pero las consideraciones aplican a cualquier régimen.

A su turno, en su rol de Tribunal Supremo de lo Contencioso Administrativo - juez por excelencia de la legalidad de los actos administrativos que concretan el ejercicio de la potestad sancionatoria de la administración-, el Consejo de Estado se ha pronunciado en pletóricas oportunidades, en alrededor de 40 providencias dictadas entre 2001 y 2018, sobre varios de los elementos que permiten comprender cómo funciona el régimen sancionatorio que regenta la Superintendencia de Servicios Públicos Domiciliarios.

En tales providencias — que no se enlistan en este párrafo en eras de la brevedad, pero que están debidamente referenciadas al final del documento- se ocupó, entre muchos otros, de temas como: 1) la competencia de la Superintendencia de Servicios Públicos Domiciliarios para imponer sanciones a las empresas prestadoras de servicios públicos domiciliarios; 2) incluso a través de los superintendentes delegados; 3) en el marco de la inspección, vigilancia y control; 4) el concepto y los componentes de las diversas modalidades de servicios públicos -electricidad, acueducto, gas, etcétera-; 5) los elementos que integran la tarifa cobrada los usuarios y 6) la definición de este grupo poblacional; 7) la caducidad de la facultad sancionatoria de la Superservicios; 8) la participación de las Comisiones de Regulación —v. gr. agua potable y saneamiento básico, comunicaciones, energía- en el proceso sancionatorio en cuestión; 9) la finalidad correctiva de la sanción; 10) el abuso de posición dominante, la modificación unilateral de tarifas, revisión 
quinquenal de acometidas, elusión de gravámenes, interrupción del suministro, entre otros aspectos sancionables; 11) el principio de legalidad y la 12) gradualidad de las sanciones; 13) las funciones administrativas de protección al consumidor, junto con 14) el procedimiento para quejas y reclamos; 15) la participación de la Superintendencia de Industria y Comercio en el modelo sancionatorio; 16) los elementos del debido proceso administrativo sancionatorio frente a las EsP; 17) los recursos procedentes contra las sanciones de la Superservicios; 18) el principio de congruencia entre la imputación y la sanción; y 19) el silencio administrativo positivo como sanción.

\section{Aproximación doctrinal a los postulados del procedimiento administrativo sancionatorio aplicado a las empresas de servicios públicos domiciliarios}

La mayoría de aportes frente al procedimiento administrativo sancionatorio aplicado a las empresas de servicios públicos domiciliarios se presentan a manera de monografías. Al nivel sociojurídico, pueden definirse los servicios públicos domiciliarios como una categoría especial de los servicios públicos. Se prestan en forma universal, continua, eficiente, obligatoria y en igualdad de condiciones y calidad a todos los usuarios, por medio de redes físicas o humanas en su sitio de habitación o trabajo, con un régimen jurídico especial de derecho público, con la prestación directa de la Administración Pública, con su regulación y control (Matías, 2015).

Es claro que la Ley 142 de 1994, en su artículo primero definió qué se entendía como servicios públicos domiciliarios, los cuales hacen parte de los servicios públicos, pero se les dio una categoría especial, denominados esenciales; estableciendo, según se anticipó, un órgano especial para su vigilancia, que es la Superintendencia de Servicios Públicos Domiciliarios, por ende, todas las actividades encaminadas al bienestar de las personas están catalogadas como servicios públicos, y el Estado, a través de los diferentes órganos de la administración, debe garantizar que estos se creen y se materialicen de manera eficiente y continua sin ser una carga para los asociados (Maldonado, 2010).

De allí la idea de la inspección, vigilancia y eficiencia como principios característicos de la Ley 142 de 1994, que determinaron la existencia de un modelo de seguimiento especial para quienes ejercen esta actividad en nombre o bajo la 
supervisión del Estado, debiendo estas empresas garantizar a los usuarios la mejor prestación de los servicios a su cargo (Palacios, 2005); siendo esta actividad un tema sumamente delicado y sensible, que involucra a sectores tanto públicos como privados.

Dentro de este tipo de literatura también se encuentra el trabajo de Sarmiento y Sarmiento (Universidad Libre, 2012) que buscó identificar si se desconoce el marco supraconstitucional en la materia al permitirse un desarrollo legal en Colombia que comprende sanciones pecuniarias y cambios de naturaleza jurídica. Allí se identificó que los estamentos con injerencia en el régimen de servicios públicos domiciliarios se encuentran ante la disyuntiva de aplicar, de un lado, el derecho convencional en defensa de los usuarios del servicio, impidiendo la deslegalización de sus principales características y la imposición de gastos desbordados en aplicación de tratados de derechos humanos ratificados por Colombia o, de otro lado, de aplicar las políticas de sostenibilidad financiera, en el marco de recuperación de un servicio que preserve otras necesidades básicas. Igualmente, dicha investigación aporta un estudio de la viabilidad de la potestad sancionatoria, desde la óptica de la Corte Constitucional, aplicada al escenario de los servicios públicos domiciliarios.

Por otra parte, Suárez (2014) investigó las tensiones constitucionales derivadas del procedimiento administrativo sancionatorio en el marco del derecho urbano en Colombia. Si bien el tema no tiene una conexión directa con el régimen jurídico de los servicios públicos domiciliarios, permite una aproximación general al procedimiento administrativo sancionatorio que ha sido introducido con la Ley 1437 de 2011, por cuanto su investigación académica describe el vínculo que existe entre el procedimiento sancionatorio disciplinario y el procedimiento administrativo sancionatorio; aunado a que también realiza precisiones en relación con diversas clases de procedimientos especiales que cuentan con su propia regulación: ambientales (Ley 1333 de 2009), urbanísticos (Ley 388 de 1997), tributarios (Decreto 624 de 1989), cambiarios (Decreto 2245 de 2011), bursátiles (Ley 964 de 2005), financieros (Ley 795 de 2003), deportivos (Decreto Ley 1228 de 1995), protección de datos personales (Ley 1581 del 2012), y protección del consumidor (Ley 1480 de 2011), entre otros, que también han sido referenciados por Suárez, Mejía y Restrepo (2014).

Bajo similares glosas, se pone de relieve un estudio de la Universidad del Norte (2018) sobre el procedimiento administrativo sancionatorio aplicado en el régimen de salud, que acomete la normativa administrativa sancionadora aplicada por la 
Superintendencia Nacional de Salud a partir de los avances de la jurisprudencia constitucional y contenciosa. Como resultado del mismo, se advirtieron falencias generales del procedimiento aplicado, que podrían hacerse extensivas al ámbito de los servicios públicos domiciliarios, y se precisaron los principios aplicables y su evolución.

Así también en un estudio realizado por Tamayo, Mejía y Restrepo (2014) se enumera buena parte de la legislación expedida entre 1991 y 2011, que contiene más de 97 procedimientos administrativos sancionatorios regulados por leyes especiales, identificándose en dicho análisis la falta de unificación de criterios acerca de principios aplicables a dichos procedimientos y etapas de este, y la forma en que la expedición de la Ley 1437 de 2011 impacta este fenómeno, especialmente en cuanto a la aplicación del principio de legalidad de las faltas (Daza, 2013).

Como ya se ha referido, existen múltiples procedimientos administrativos sancionatorios, a falta de unificación normativa en la materia, que generan constantes variaciones en cada uno de ellos. Un claro ejemplo es la Ley 1333 de 2009 en materia de regulación ambiental, que sufrió cambios rituales en razón del trámite sancionatorio general que introdujo la Ley 1437 de 2011, que implicó que, en aras de respetar el debido proceso, se introdujeran cambios en la comunicación a terceros interesados, así como en el traslado para alegar y el traslado de las pruebas aportadas con el recurso de reposición (Garro, 2013). Ahora, hay que decir, sin temor a incurrir en tautología, que en el ámbito de servicios públicos domiciliarios no existe un procedimiento sancionatorio administrativo especial, es decir, se rige íntegramente por la Ley 1437 de 2011 (García y Fino, 2014).

Frente a su caracterización, huelga indicar que, con un enfoque similar al de otros autores mencionados líneas atrás, Nettel y Rodríguez (2018) publicaron un artículo en la Revista Misión Jurídica de Derecho y Ciencias Sociales, de Bogotá, que abordó un aspecto axiológico más amplio, que incluye la caracterización del debido proceso y la presunción de inocencia, desde un análisis de casos (tutelas), enfatizando los matices penales del procedimiento administrativo sancionador y descartando que ello pueda condicionar el análisis de sus diferencias.

De otra suerte, Carvajal (2010), en la revista digital de Derecho Administrativo, examina el alcance y limitaciones del debido proceso en el procedimiento administrativo y su relación con los principios de favorabilidad, non bis in ídem, presunción de inocencia y el ejercicio del derecho de defensa en el campo administrativo 
sancionatorio, lo cual, según desarrolla, debe acompasarse con la prohibición de dilaciones injustificadas y las garantías probatorias propias del derecho colombiano.

$\mathrm{Y}$ es que en este contexto es importante destacar como primer postulado el respeto al debido proceso en las actuaciones administrativas que ejerce dicha entidad, para ello, no es de poca importancia resaltar su connotación constitucional, pues se trata nada más y nada menos que de un derecho fundamental, que, a su vez, incorpora una serie de garantías procesales, tales como la publicidad, el derecho de defensa, las necesidad de reglas probatorias claras, la presunción de inocencia, el derecho a presentar impugnaciones, objeciones y recursos, entre otras (Carvajal, 2010), que analizadas en conjunto le dan un sentido constante y permanente, vinculado a la idea de justicia o equidad procesal. Así, una actuación administrativa sancionatoria que desconozca estos parámetros iría en contravía del Estado social de derecho (Palomares, 2015).

También se cuenta con el aporte realizado Lozano y Castro (2013), a través de la revista digital de Derecho Administrativo de la Fundación Universitaria Los Libertadores, en el que se analizó la facultad sancionatoria de las empresas de servicios públicos domiciliaros en materia de energía en Colombia. Dichos autores detectaron un vacío legal que ha afectado los intereses económicos por el pago de las sanciones pecuniarias impuestas por aquellas a sus usuarios. De su aporte también se destaca la caracterización de los denominados procedimientos para la recuperación de consumos, diferentes al ejercicio de imposición de una sanción. Para ello se valen de un análisis de la Sentencia su-1010 de 2008, que conduce a la ausencia de fundamentos normativos para el ejercicio de tal atribución por parte de las prestadoras, en contraste con las facultadas para el cobro de consumos dejados de facturar a causa de conexiones irregularmente constituidas (Rodríguez, 2016).

Igualmente, debe decirse que Zárate (2018) publicó un artículo sobre el poder sancionatorio de la administración pública, en el cual se abordó el tema de la facultad sancionatoria de la Superintendencia de Servicios Públicos Domiciliarios frente a las empresas de servicios públicos, y se determinó que, al lado del procedimiento administrativo sancionatorio del cPACA aplicable a las actuaciones que se adelantan para investigar y eventualmente sancionar a dichas empresas y personas naturales que infringen las normas del régimen de los servicios públicos domiciliarios coexiste un procedimiento especial para otro tipo de medidas que no tienen el alcance de sanción (Guarín y Aldana, 2016). 
Para finalizar, cabe advertir que en materia de los servicios públicos domiciliarios han cobrado relevancia los conceptos emitidos por la Superintendencia de Servicios Públicos Domicilios, como una forma de impetrar y suplir de alguna manera los vacíos de la Ley 142 de 1994 y demás referentes normativos, destacando el rol protagónico, como solución eficiente, que deben tener las autoridades públicas cuando no se cumple con dicha correspondencia (Arocha, 2013), y optando por señalar en muchos conceptos unificadores el debido proceso que deben cumplir las empresas prestadoras de servicios públicos en algunas actuaciones a su cargo, con el fin de no violar el debido proceso.

\section{Conclusiones}

El procedimiento administrativo sancionatorio, de manera general, ha venido siendo regulado de manera dispersa para los diferentes sectores administrativos, prueba de ello son los múltiples procedimientos sancionatorios que han sido impuestos para diferentes sectores, a falta de un procedimiento general. Es claro que a nivel general esa dispersión normativa hace que muchas veces los ciudadanos y las empresas no conozcan sus obligaciones y las incumplan, lo cual redunda en un mayor número de sancionados.

Si bien la Ley 1437 de 2011 establece de manera general un procedimiento administrativo sancionatorio, no sustituyó o eliminó aquellos procedimientos especiales, motivo por el cual actualmente se hace complejo determinar si una conducta es sujeta de sanción o no y de qué manera deben ponderarse los insumos cognoscitivos que permiten su imposición.

El régimen administrativo sancionatorio consagrado en la Ley 1437 de 2011 aplicado a las empresas de servicios públicos domiciliarios, si bien brindó una organización general del procedimiento que la Superintendencia de Servicios Públicos Domiciliarios ejerce para sancionar a las empresas bajo su inspección, vigilancia y control, aun no parece garantizar el cumplimiento de todos los postulados base de un procedimiento administrativo sancionatorio; por lo cual todo apunta a que se requiere un procedimiento especial que contemple los diferentes aspectos con miras a garantizar el debido proceso y los principios que le son inherentes, especialmente la necesidad de que exista una gradualidad de las sanciones que permita anteponer el derecho de defensa y contradicción en estos complejos escenarios 
El procedimiento administrativo sancionatorio en el régimen de servicios públicos domiciliarios tiene naturaleza jurídica con un claro raigambre constitucional, cuyo actual desarrollo se encuentra enfocado a la aplicación al procedimiento general de la Ley 1437 de 2011 que ha venido acondicionándose a partir de los aportes de la jurisprudencia de la Corte Constitucional y el Consejo de Estado.

Los postulados básicos del proceso administrativo sancionatorio en el marco del régimen de Servicios Públicos Domiciliarios son los señalados en el procedimiento administrativo general consagrado en la Ley 1437 de 2011, sin embargo, no se encuentra un desarrollo jurídico amplio de los principios de gradualidad y de proporcionalidad de las sanciones.

La Ley 142 de 1994 no determinó un procedimiento para dichas actuaciones, de manera tal que, luego de proferida esta ley, únicamente se había desarrollado un proceso administrativo sancionatorio acudiendo a las normas de carácter general que existían en el anterior código contencioso; empero, con la expedición de la Ley 1437 de 2011 se empezó a dar aplicación al procedimiento en ella regulado, aunque este procedimiento, como bien se ha denominado, es general y existen ámbitos propios de la naturaleza de los servicios públicos que no se encuentran regulados, como la proporcionalidad de las sanciones, de acuerdo con su clasificación como graves, leves o levísimas; aunado a que existe una falta de tipificación de los actos por sancionar, lo que amplía la esfera de la discrecionalidad de la administración al momento de ejercer dicha facultad.

Con el análisis de los planes de desarrollo, se ha evidenciado el interés del ejecutivo de aumentar los montos de las sanciones y ampliar el valor pecuniario de estas, lo que no debería corresponder a un simple análisis de recaudos, sino que debería desarrollarse dentro de cada ámbito a sancionar el impacto que ha generado la facultad sancionadora, si esta está cumpliendo con el fin de que las empresas de servicios públicos mejoren sus procedimientos de atención a los usuarios, o si, por el contrario, los prestadores de servicios públicos ya se han acostumbrado a las sanciones y no mejoran su actividad.

\section{Referencias}

Aldana, J. y Guarín, E. (2016). Los límites de la teoría del equilibrio como alternativa de solución a la cuestión de la prevalencia del poder del Alto Tribunal de lo Constitucional en Colombia. Revista Via Inveniendi et Iudicandi, 11(1), 59-82. Documento extraído 
el 3 de marzo de 2018 de http://revistas.usantotomas.edu.co/index.php/viei/article/ view/2923/2796

Arocha, R.N. (2013). Los conceptos unificadores como propuesta institucional ante los eventos de disciplina autista. Revista Universitas Estudiantes, 10, 11-36. Universidad Javeriana.

Carvajal, B. (2010). Alcance y limitaciones del debido proceso en el procedimiento administrativo. Revista digital de Derecho Administrativo, 4, 7-21.

Colombia. Decreto 1077 de 2015. Por medio del cual se expide el Decreto Único Reglamentario del Sector Vivienda, Ciudad y Territorio. Mayo 26 de 2015. D. O. núm. 49.523.

Colombia. Decreto 1082 de 2015. Por medio del cual se expide el decreto único reglamentario del sector administrativo de planeación nacional. Mayo 26 de 2015. D. O. núm. 49523.

Colombia. Decreto 1158 de 2017. Por el cual se adiciona el Decreto 1082 de 2015, con el fin de reglamentar los criterios y metodología para graduar y calcular las multas por parte de la Superintendencia de Servicios Públicos Domiciliarios por infracciones relacionadas con los servicios públicos domiciliarios de acueducto, alcantarillado y aseo. Julio 7 de 2017. D. O. núm. 50.287.

Colombia. Decreto 2245 de 2011. Por el cual se establece el Régimen Sancionatorio y el Procedimiento Administrativo Cambiario a seguir por la Dirección de Impuestos y Aduanas Nacionales. Junio 28 de 2011. D. O. núm. 48114.

Colombia. Decreto 281 de 2017. Por el cual se adiciona el Decreto 1082 de 2015, con el fin de reglamentar los criterios y metodología para graduar y calcular las multas por parte de la Superintendencia de Servicios Públicos Domiciliarios, por infracciones relacionadas con el servicio de energía eléctrica. Febrero 22 de 2017. D. O. núm. 50.155.

Colombia. Decreto 624 de 1989. Por el cual se expide el Estatuto Tributario de los impuestos administrados por la Dirección General de Impuesto Nacionales. Marzo 30 de 1989. D. O. núm. 38.756.

Colombia. Decreto Ley 1228 de 1995. Por el cual se revisa la legislación deportiva vigente y la estructura de los organismos del sector asociado con objeto de adecuarlas al contenido de la Ley 181 de 1995. Julio 18 de 1995. D. O. núm. 41.933.

Colombia. Ley 142 de 1994. Por la cual se establece el régimen de los servicios públicos domiciliarios y se dictan otras disposiciones. Julio 11 de 1994. D. O. núm. 41.433.

Colombia. Ley 1437 de 2011. Por la cual se expide el Código de Procedimiento Administrativo y de lo Contencioso Administrativo. Enero 18 de 2011. D. O. núm. 47.956. 
Colombia. Ley 1480 de 2011. Por medio de la cual se expide el Estatuto del Consumidor y se dictan otras disposiciones. Octubre 12 de 2011. D. O. núm. 48.220.

Colombia. Ley 1581 del 2012. Por la cual se dictan disposiciones generales para la protección de datos personales. Octubre 17 de 2012. D. O. núm. 48.587.

Colombia. Ley 1753 de 2015. Por la cual se expide el Plan Nacional de Desarrollo 20142018 “Todos por un nuevo país”. Junio 9 de 2015. D. O. núm. 49.538.

Colombia. Ley 388 de 1997. Por la cual se modifica la Ley 9a de 1989, y la Ley 3a de 1991 y se dictan otras disposiciones. Julio 18. D. O. núm. 43.091.

Colombia. Ley 795 de 2003. Por la cual se ajustan algunas normas del Estatuto Orgánico del Sistema Financiero y se dictan otras disposiciones. Enero 14 de 2003. D. O. núm. 45.064.

Colombia. Ley 964 de 2005. Por la cual se dictan normas generales y se señalan en ellas los objetivos y criterios a los cuales debe sujetarse el Gobierno Nacional para regular las actividades de manejo, aprovechamiento e inversión de recursos captados del público que se efectúen mediante valores y se dictan otras disposiciones. Julio 8 de 2005. D. O. núm. 45.963.

Colombia. Ley 1333 de 2009. Por la cual se establece el procedimiento sancionatorio ambiental y se dictan otras disposiciones. Julio 21 de 2009. D. O. núm. 47.417.

Corte Constitucional de Colombia [C. C.], abril 28 de 1994, M. P.: A. J. Barrera, Sentencia C-214/94, [Colombia].

Corte Constitucional de Colombia [C.C.], agosto 9 de 2005, M. P.: R Escobar, Sentencia C-818/05, [Colombia].

Corte Constitucional de Colombia [C. C.], julio 27 de 2010, M. P.: J. I. Palacio, Sentencia C-595/10, [Colombia].

Corte Constitucional de Colombia [C. C.], mayo 31de 2001, M. P.: J. Araujo, Sentencia C-558/01, [Colombia].

Corte Constitucional de Colombia [C. C.], octubre 16 de 2008, M. P.: R. Escobar, Sentencia SU-1010/08, [Colombia].

Corte Constitucional de Colombia [C. C.], octubre 3 de 2018, M. P.: A. Rojas, Sentencia C-092/18, [Colombia].

Consejo de Estado [C.E.], Sala Plena, agosto 9 de 2016, C. P.: W. Hernández, Sentencia 2011-00316/121011, [Colombia].

Consejo de Estado [C. E.], Sala Plena, marzo 5 de 2014, C. P.: A. Vargas, Sentencia 201306871, [Colombia]. 
Consejo de Estado [C.E.], Sección Cuarta, agosto 30 de 2016, C. P.: H. F. Bastidas, Sentencia 2008-01220/19851, [Colombia].

Consejo de Estado [C.E.], Sección Primera, abril 27 de 2001, C. P.: M. S. Urueta, Sentencia 1999-00001, [Colombia].

Consejo de Estado [C.E.], Sección Primera, agosto 18 de 2011, C. P.: M. C. Rojas, Sentencia 2002-00911, [Colombia].

Consejo de Estado [C.E.], Sección Primera, agosto 23 de 2012, C. P.: M. E. García, Sentencia 2004-01001, [Colombia].

Consejo de Estado [C. E.], Sección Primera, agosto 4 de 2011, C. P.: M. C. Rojas, Sentencia 2003-01151, [Colombia].

Consejo de Estado [C.E.], Sección Primera, agosto 6 de 2004, C. P.: C. Arciniegas, Sentencia 2001-00033, [Colombia].

Consejo de Estado [C.E.], Sección Primera, agosto 9 de 2007, C. P.: R. E. Osteau de Lafont, Sentencia 2001-00321, [Colombia].

Consejo de Estado [C.E.], Sección Primera, diciembre 13 de 2001, C. P.: M. A. Velilla, Sentencia 2001-00015, [Colombia].

Consejo de Estado [C.E.], Sección Primera, enero 23 de 2014, C. P.: M. A. Velilla, Sentencia 2004-00633, [Colombia].

Consejo de Estado [C.E.], Sección Primera, febrero 14 de 2013, C. P.: M. A. Velilla, Sentencia 2003-91003, [Colombia].

Consejo de Estado [C.E.], Sección Primera, febrero 23 de 2012, C. P.: M. E. García, Sentencia 2004-00344, [Colombia].

Consejo de Estado [C. E.], Sección Primera, julio 3 de 2014, C. P.: M. E. García, Sentencia 2010-00666, [Colombia].

Consejo de Estado [C.E.], Sección Primera, noviembre 29 de 2001, C. P.: M. S. Urueta, Sentencia 1999-00167, [Colombia].

Consejo de Estado [C.E.], Sección Primera, noviembre 6 de 2014, C. P.: M. C. Rojas, Sentencia 2006-00321, [Colombia].

Consejo de Estado [C.E.], Sección Primera, octubre 10 de 2012, C. P.: M. A. Velilla, Sentencia 2004-00523, [Colombia].

Consejo de Estado [C.E.], Sección Primera, octubre 20 de 2019, C. P.: O. Giraldo, Sentencia 2009-00408, [Colombia]. 
Consejo de Estado [C.E.], Sección Primera, octubre 22 de 2004, C. P.: O. I. Navarrete, Sentencia 2001-01132, [Colombia].

Consejo de Estado [C.E.], Sección Primera, octubre 22 de 2015, C. P.: R. A. Serrato, Sentencia 2010-00654, [Colombia].

Consejo de Estado [C.E.], Sección Primera, septiembre 10 de 2015, C. P.: R. A. Serrato, Sentencia 2006-00025, [Colombia].

Consejo de Estado [C.E.], Sección Primera, septiembre 18 de 2014, C. P.: G. Vargas, Sentencia 2008-00282, [Colombia].

Consejo de Estado [C.E.], Sección Primera, septiembre 20 de 2002, C. P.: C. Arciniegas, Sentencia 1999-00250, [Colombia].

Consejo de Estado [C.E.], Sección Primera, septiembre 29 de 2019, C. P.: M. C. Rojas, Sentencia 2004-00370, [Colombia].

Consejo de Estado [C.E.], Sección Primera, septiembre 30 de 2010, C. P.: R. E. Osteau De Lafont, Sentencia 2007-00203, [Colombia].

Consejo de Estado [C.E.], Sección Quinta, abril 12 de 2018, C. P.: C. E. Moreno, Sentencia 2008-00198, [Colombia].

Consejo de Estado [C.E.], Sección Quinta, abril 18 de 2018, C. P.: R. Araújo, Sentencia 2006-01180, [Colombia].

Consejo de Estado [C.E.], Sección Quinta, agosto 2 de 2018, C. P.: A. Yepes, Sentencia 2011-00450, [Colombia].

Consejo de Estado [C.E.], Sección Quinta, febrero 15 de 2018, C. P.: C. E. Moreno, Sentencia 2008-00509, [Colombia].

Consejo de Estado [C.E.], Sección Quinta, febrero 22 de 2018, C. P.: R. Araújo, Sentencia 2010-00348, [Colombia].

Consejo de Estado [C.E.], Sección Quinta, julio 12 de 2018, C. P.: A. Yepes, Sentencia 2007-00497, [Colombia].

Consejo de Estado [C.E.], Sección Quinta, julio 12 de 2018, C. P.: C. E. Moreno, Sentencia 2012-00399, [Colombia].

Consejo de Estado [C.E.], Sección Quinta, julio 12 de 2018, C. P.: L. J. Bermúdez, Sentencia 2009-00307, [Colombia].

Consejo de Estado [C.E.], Sección Quinta, mayo 10 de 2018, C. P.: R. Araújo, Sentencia 2008-00215, [Colombia 
Consejo de Estado [C.E.], Sección Quinta, mayo 10 de 2018, C. P.: R. Araújo, Sentencia 2009-00353, [Colombia].

Consejo de Estado [C.E.], Sección Quinta, mayo 17 de 2018, C. P.: R. Araújo, Sentencia 2007-00465, [Colombia].

Consejo de Estado [C.E.], Sección Quinta, mayo 3 de 2018, C. P.: A. Yepes, Sentencia 2007-00002, [Colombia].

Consejo de Estado [C.E.], Sección Quinta, mayo 31 de 2018, C. P.: L. J. Bermúdez, Sentencia 2010-90221, [Colombia].

Consejo de Estado [C. E.], Sección Segunda, agosto 4 de 2016, C. P.: S. L. Ibarra, Sentencia 2016-00522, [Colombia

Consejo de Estado [C.E.], Sección Segunda, septiembre 7 de 2000, C. P.: A. M. Olaya, Sentencia AP-088, [Colombia].

Consejo de Estado [C.E.], Sección Tercera, junio 4 de 2015, C. P.: J. O. Santofimio, Sentencia 2001-00104, [Colombia].

Consejo de Estado [C.E.], Sección Tercera, octubre 22 de 2012, C. P.: E. Gil, Sentencia 1996-00680/20738, [Colombia].

Constitución Política de Colombia [C.P.]. Julio 7 de 1991 (Colombia).

Cordero C.R. (2009). El debido procedimiento administrativo sancionador. Revista de Derecho Público, 71, 183-214.

Cortes, S. (2013). La globalización económica y los derechos humanos. Revista Via Inveniendi et Iudicandi, 8(2), 138-149.

Daza, A. (2013). Legalidad y prescripción frente a la investigación de crímenes de lesa humanidad en Colombia. Revista IUSTA, 38(1), 205-223.

De La Cruz, A. (2018). Análisis de la normativa administrativa sancionadora aplicada por la Superintendencia Nacional de Salud a partir de los principios del DAs desarrollados por la doctrina constitucional colombiana. Tesina presentada como requisito para optar al título de magíster en Derecho. Universidad del Norte. Barranquilla, Colombia.

García, M. y Fino, G. (2014). Los impuestos territoriales en Colombia y la inequidad social, ¿la voluntad de la clase dominante erigida en ley? Revista IUSTA, 41, 61-75. Recuperado el 2 de febrero de 2018 de http://revistas.usta.edu.co/index.php/iusta/ article/view/2471/2408 
Garro, A. (2013). Procedimiento administrativo sancionatorio ambiental a partir de la vigencia de la Ley 1437 de 2011. Revista Facultad de Derecho y Ciencias Politicas, 43(118), 443-470.

Guarín, E. A. y Aldana, J. (2016). Estado jurisdiccional y bien común. Revista Verba Iuris, 11(36), 13-26.

Guarín, E. (2015). Una aproximación filosófico-jurídica al sentido de la expresión: "Realización efectiva de los derechos". Revista Verba Iuris, 34, 147-159. Recuperado el 8 de mayo de 2018 de https://revistas.unilibre.edu.co/index.php/verbaiuris/article/view/16/14

Laverde, J.M. (2016). D. Juan Manuel Laverde en el Consejo de Estado [Vídeo]. Recuperado de https://youtu.be/ibKBaKdk2ys

Laverde, J. M. (2016). Manual de procedimiento administrativo sancionatorio. (1. a ed.). Bogotá D. C., Colombia: Legis.

León M.A. (2009). El principio de proporcionalidad en el procedimiento sancionador: metodología para la determinación de la sanción administrativa. Ius Et Veritas, 38, 296-310.

Lozano, D.R. y Castro, O.E. (2013). ¿Carecen de facultad sancionatoria las empresas de servicios públicos domiciliarios frente a los usuarios? Un estudio con relación al servicio de energía eléctrica en Colombia. Via Iuris, 15, 49-63.

Maldonado, T. (2010). La noción de servicio público a partir de la concepción del Estado social de derecho. Revista Actualidad Jurídica, 1, 54-62.

Matías, S.R. (2015). Los servicios públicos domiciliarios en Colombia: su prestación, regulación y control. Revista del CLAD Reforma y Democracia, 63, 163-194.

Mesa, J. C. (2016). La caracterización de la Ley 1437 de 2011, tiene contemplados los principios que se anejan del derecho penal en materia administrativa sancionatoria, como garantías de un debido proceso administrativo. Bogotá D.C., Colombia. Repositorio Universidad Santo Tomás.

Millán, J., Blanco, C. y Guecha, C. (2017). Los procesos de selección de operadores privados del servicio público de televisión en torno a la libre concurrencia en Libro Justicia Constitucional Tomo II. (pp. 199- 221). Recuperado el 2 de febrero de 2019 de https://repository.usta.edu.co/handle/11634/4249

Nettel, A. C. y Rodríguez, L. G. (2018). El derecho administrativo sancionador en el ámbito disciplinario de la función pública. Revista de Derecho y Ciencias Sociales, 14, 115-128.

Palacios, M.T. (2005). El derecho al servicio público domiciliario de acueducto. Opinión Jurídica, 4(7), 13-32. 
Palomares, J. (2015). El carácter vinculante de la jurisprudencia constitucional en el derecho alemán. Revista Via Inveniendi et Iudicandi, 10(2), 29-56. Recuperado el 3 de enero de 2018 de evistas.usantotomas.edu.co/index.php/viei/article/view/2551/2483

Pardo, N. (2014). Un recorrido por los derechos colectivos en la jurisprudencia argentina. Revista Via Inveniendi et Iudicandi, 9(1), 32-49. Recuperado el 2 de enero de 2018 de http://revistas.usantotomas.edu.co/index.php/viei/article/view/1338/1540

Ramírez, M. L. y Echeverría, J. (2009). La potestad sancionadora de las empresas de servicios públicos domiciliarios y su constitucionalidad. El caso de Colombia. Revista de Derecho de la Pontificia Universidad Católica de Valparaíso XXXIII, 429-452.

Ramírez, M. L. y Aníbal, H.V. (2015). Sanción administrativa en Colombia. Revista Vniversitas, 131, 107-148.

Restrepo, M. y Nieto, M. (2017). El derecho administrativo sancionador en Colombia. Bogotá D. C., Colombia: Editorial universidad del Rosario.

Rodríguez, E. (2016). El pasaje del estado y el derecho a la postmodernidad. Revista Via Inveniendi et Iudicandi, 11(2), 11-37.

Sarmiento, I. E. y Sarmiento, M. (2012). Marco constitucional, legal y facultad sancionatoria de las empresas de servicios públicos domiciliarios. Monografía realizada para optar el título de magíster en Derecho Administrativo. Universidad Libre. Bogotá D. C., Colombia.

Sierra, H. J. (2015). “Responsabilidad de los prestadores de servicios públicos domiciliarios en el procedimiento administrativo sancionatorio en Colombia”. Artículo de reflexión como opción de grado, Especialización en Derecho Administrativo, Universidad Militar Nueva Granada. Bogotá D. C., Colombia.

Suárez, N. C. (2014). Tensiones constitucionales derivadas del procedimiento administrativo sancionatorio en el marco del derecho urbano en Colombia. Monografía para obtener grado de Maestría en Derecho Administrativo Universidad Libre. Bogotá C.D., Colombia.

Suárez, D., Mejía, P. y Restrepo, L. (2014). Procedimientos administrativos sancionatorios Inventario normativo y de las sentencias de la Corte Constitucional de Colombia. Opinión Jurídica, 13(25), 139-154.

Zárate, P.A. (2018). La especialidad relativa de la potestad sancionadora en materia de servicios públicos domiciliarios. Bogotá D. C., Colombia: Universidad Externado de Colombia. 775-852. 